\title{
Research on Calculation Method of Total Solar Radiation of Urban Settlements Based on Parametric 3D Model
}

\author{
Ruixin Chen ${ }^{1,2}$, Yan Liu ${ }^{1,2 *}$, Moyan Zhang ${ }^{1,2}$, Qi Li ${ }^{1,2}$, Hao Zhang ${ }^{1,2}$, Liu Yang ${ }^{1,2}$ \\ ${ }^{1}$ State Key Laboratory of Green Building in Western China, Xi' an 710055, P.R. China \\ ${ }^{2}$ School of Architecture, Xi' an University of Architecture and Technology, Xi' an 710055, P.R. China
}

\begin{abstract}
Solar radiation is a major factor in promoting the sustainable development of urban settlements. To understand the distribution of solar radiation in urban settlements, this paper develops the sky view factor (SVF) algorithm based on the parameterization platform to simplify the calculation of solar radiation. In this paper, the test of total solar radiation is carried out in 18 different locations in Xi'an University of Architecture and Technology. The total solar radiation of 18 points is calculated using the SVF-based calculation model. Comparing the calculated results with the measured results, it is found that the calculated values are consistent with the measured values. The results show that the algorithm is consistent with the measured data. It shows the effectiveness and high precision of the proposed algorithm. The algorithm can calculate the total solar radiation more quickly, and provide technical support for urban preliminary design schemes in urban microclimate.
\end{abstract}

\section{Introduction}

With more than the half of the world population living in cities today [1], urban environmental sustainability has become the frame researchers and practitioners in urban design and planning. Solar radiation is a major factor in promoting environmental sustainability in urban settlements. Due directionality of solar rays and predictable interactions with urban geometries, solar exposure on urban surfaces can be accurately modeled [2]. To understand the distribution of solar radiation in urban settlements will help provide technical support for the city's project design.

Among the factors affecting solar radiation in urban settlements, urban form is an important factor affecting solar radiation in urban settlements. sky occlusion refers to the occlusion of the sky seen anywhere on the surface by surrounding buildings, trees or other structures. Due to sky occlusion, the solar radiation received by the urban ground is different from that of the suburbs, which in turn affects the near-surface temperature and the urban heat island intensity. The presence of sky occlusion also affects the height average radiant temperature of the pedestrian and the thermal comfort of the human body, as well as the heating and energy consumption of the building [3]. The sky view factor (SVF) is a morphological parameter that describes the degree of occlusion of the sky and is a key parameter for calculating surface radiation [4].

Now, more and more solar and thermal analysis models perform accurate SVF calculations across the city's surface and at different spatial resolutions as part of their simulation. SVF is much faster to calculate than solar radiation simulation [4]. In the urban residential area, due to the complicated construction layout, the computational difficulty of SVF is increased. In order to simplify the calculation difficulty of SVF and improve the calculation speed, the main work of this paper is as follows. (1) The development parametric model of the Rhino-Grasshopper (RH \& GH) platform based on the Ladybug plugin to realize the automatic calculation of SVF. (2) The solar radiation calculation model based on SVF is selected to calculate the total solar radiation value at a certain point on the ground. (3) The calculation accuracy, reliability and operation of the algorithm are verified by comparing the calculated data with the actual test data of the settlement effectively.

\section{Methodology}

This paper implements the automatic calculation of SVF based on the parametric 3D model plug-in platform, selects the solar radiation calculation based on SVF, and compares the calculated data with the test data in order to test the accuracy of the algorithm and the calculation model.

\subsection{SVF calculation}

The SVF, derived from the concept of "view factor", is "a geometric ratio that represents the portion of a surface whose radiant output is intercepted by another surface." There are two different definitions in different background studies. For researchers in architecture and urban design, SVF is defined as the proportion of sky visible from a point to the entire sky dome [5,6]. SVF is

\footnotetext{
*Corresponding author: liuyan@xauat.edu.cn
} 
based on the assumption that the various parts of the sky are equally important. The SVF is calculated as the ratio of the solid angle of the sky plaque visible from a certain point to the solid angle of the hemisphere centered at the same point, and is named as the geometric definition of SVF. The SVF estimation method can be divided into the following four categories: analysis method [3, 7, 8], fisheye camera technology $[9,10]$, GPS signal estimation [11], and estimation using 3D buildings $[12,13]$.

For the three-dimensional digital model, Li et al. [14] methods based on the sky-to-ground reverse analysis method developed the corresponding urban SVF calculation model. Wu et al. [15] based on the occlusion angle algorithm and the inverse trigonometric function analysis method is directly used scripting language to calculate SVF in RH \& GH. Based on the above situation, this paper develops a more convenient SVF automatic calculation method based on RH \& GH parameterization platform and Ladybug plug-in.

\subsection{Parametric algorithm plug-in development}

Based on the RH \& GH platform, the script development parametric algorithm plugin is written with the Ladybug plugin and the Python language. Ladybug is an open source environment plugin for Grasshopper. It works with the Energyplus weather file (ewp) to output a series of visualizations and simple calculations [16]. Description of the Shading Mask in the Ladybug plugin. Use this component to see the portion of the sky dome that is covered by the environment geometry around a given surface, which will create a separate mesh for the masked and visible parts of the sky. The component will also calculate the percentage of the sky and the visible percentage (sky view factor) that is covered by the context geometry. This paper uses this component, the specific process is described as follows: (1) By putting the Ladybug component into the GRAS through the parameterization module, (2) Importing an Energy plus weather file (epw), you can import from the Internet address [17] (3) Connect Sun-path to the location, date, time, sun-path scale set a specific value. (4). Access the Ladybug-Shading Mask to the center Pt Or Plane to access the measurement point, select the building group for the context, select the number for the sky Density, and then output the result for the Sky Exposure connection. Based on this process, the RH \& GH platform realizes the functional integration of 3D modeling and simplified calculation, and completes the SVF automatic calculation of the parametric model.

\subsection{Test}

The Xi'an University of Architecture and Technology Campus faculty and staff was selected to test the solar radiation for the test site. Since the modeling of the trees was not accurate for the Rhino modeling software, the new residential area with few trees was selected as the test site. The test instrument is the JTR05T solar total radiometer (Fig. 1). The test time is August 10, 2019, to August 12, 2019. The weather is in good condition and there is no cloud on sunny days. The daily test time is from 09:00 to 18:00. The settlement consists of 34 individual buildings with a single building height of $54 \mathrm{~m}$ and $33 \mathrm{~m}$. In order to consider the influence of different layout patterns on the test results, a total of 18 measuring points (Fig. 2) were selected, and the test height was $1.5 \mathrm{~m}$ from the ground.

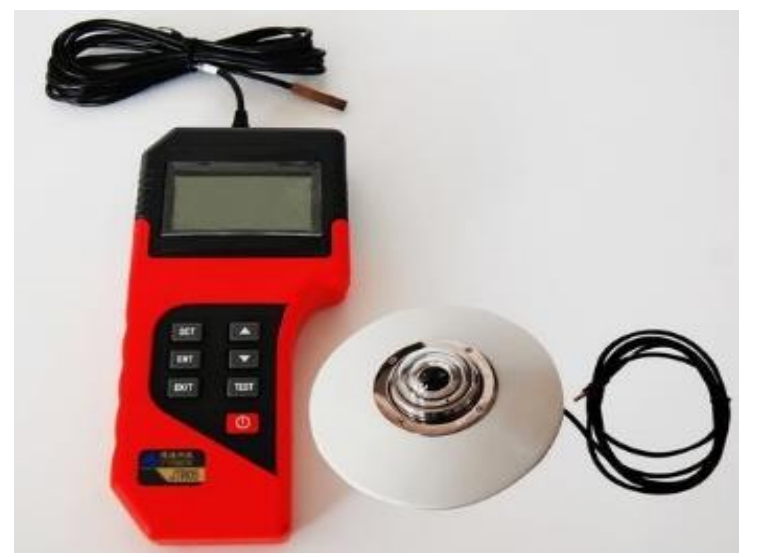

Fig.1. Solar radiation test instrument.

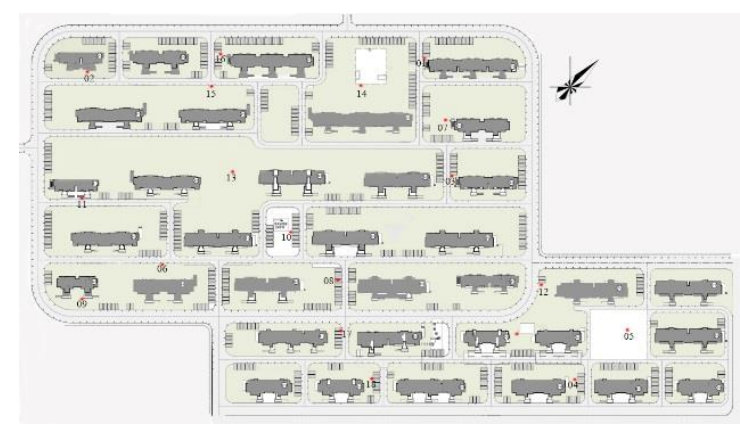

Fig.2. Arrangement of measuring points on the plan.

\subsection{Solar radiation calculation model}

This paper uses the calculated solar radiation model Zhang [18] used in his paper. In order to calculate solar radiation at any point on the ground, some basic astronomical parameters must be established, including solar height and azimuth, solar declination, and solar radiation intensity at normal incidence in the upper atmosphere. The calculation model is shown in equations (1)-(12).

\subsubsection{Astronomical parameters}

The solar declination is the angle between the line connecting the center of the earth and the center of the sun and the plane of the Earth's equator. In this study, the formula proposed by Zuo [19] is used to calculate the solar declination. Based on the function of $b$, this article will not go into details.

$$
\mathrm{b}=2 * \pi *(\mathrm{~N}-1) / 365
$$

where $\mathrm{N}$ is the number of Confucian calendar days. The $\mathrm{N}$ in the test date are 221, 222, and 223, respectively. 
The angle of time is the angle of rotation of the Earth in the unit. It is used to describe the movement of the sun within 24 hours. which is:

$$
h_{s}=15 *(h-12)
$$

where $h$ is the calculation time. During the test time, the angle is in the range of $-45^{\circ}$ to $90^{\circ}$, increasing by $15^{\circ}$ per hour.

\subsubsection{Solar Height Angle}

The solar elevation angle is the angle between the sun's rays and the horizontal plane. It changes continuously throughout the day. Related to geographic latitude, solar declination, and time angle. Calculation formula for the solar elevation angle [20]:

$$
\sin \alpha=\sin L * \sin \varphi+\cos L * \cos \varphi * \cos h_{s}
$$

where $\alpha$ is the solar elevation angle, ranging from $-90^{\circ}$ to $90^{\circ}$; $\mathrm{L}$ is the geographic latitude, and $\varphi$ is the latitude of each measurement point can be obtained by Google Earth; $\varphi$ is the sun declination; $\varphi$ Calculated by the Zuo formula, the result is $0.275734928 、 0.27064643$ and 0.26475256 respectively in text day.

$h_{s}$ is the time angle.

\subsubsection{Vertical incident solar intensity in the upper atmosphere}

Due to the solar distance factor, the solar radiation intensity does not occur in the atmosphere. Therefore, the solar radiation distance correction coefficient is introduced to correct the vertical incident solar radiation intensity in the upper atmosphere. Its formula is [19]:

$$
I_{0}=S_{0} *\left(\frac{1}{p}\right)^{2}
$$

where $\left(\frac{1}{p}\right)^{2}$ is the correction coefficient of solar and land distance, the solution can be determined according to the literature [21], and it is related to $b$. This paper directly gives the correction coefficient within three days of the test. $\mathrm{S}_{0}$ is the solar constant, which is the average distance between the sun and the earth. The outer layer of the atmosphere receives the radiant energy perpendicular to the solar ray per unit area per unit time. The value of this parameter is $1367 \mathrm{~W} / \mathrm{m}^{2}$.

\subsubsection{Calculation of direct solar radiation}

The calculation of direct solar radiation on the ground requires first obtaining the atmospheric transmittance of direct radiation. In this paper, Kreith and Kreidert [22] calculate the atmospheric transmittance of direct radiation using the empirical equation for calculating the atmospheric transmittance in the case of clear cloudless weather. The formula is:

$$
\tau_{b}=0.56 *\left(e^{-0.56 * M_{h}}+e^{-0.095 * M_{h}}\right)
$$

where $M_{h}$ is the amount of atmosphere at a certain terrain height. The atmospheric amount is a function of the solar elevation angle, the terrain height, and the atmospheric pressure. The terrain height can be obtained through Google Earth.

Based on the known direct radiation atmospheric transmittance and solar elevation angle, vertical direct solar radiation can be calculated.

$$
\mathrm{I}_{\mathrm{b}}=\mathrm{I}_{0} * \tau_{b} * \sin \alpha
$$

where $I_{b}$ is vertical ground direct radiation

\subsubsection{Calculation of Solar Diffuseing Radiation}

This paper uses a zhang [18] model based on the Iqbal C model, a simplified model to calculate the amount of solar radiation reaching the ground, independent of water vapor and aerosol. This amount of radiation is caused by the molecular diffusion $\mathrm{Dr}$ and the multiple diffusion Dm between the Earth's surface and the atmosphere. Taking into account the occlusion of the building, the sky factor SVF parameters were added to estimate the solar diffuse radiation reaching the street surface [23], which is calculated as:

$$
I_{d}=\left(D_{r}+D_{m}\right) * S V F
$$

where, $I_{d}$ is the solar diffuse radiation reaching the ground. The calculation formulas of $D_{r}$ and $D_{m}$ are as follows:

$$
\begin{aligned}
& D_{r}=0.79 * I_{0} * \sin \alpha * \tau_{0} * \tau_{g} *\left(1-\tau_{r}\right) /\left(1-M_{h}+\right. \\
& \left.M_{h}^{1.02}\right) \\
& \quad D_{m}=\left(I_{b}+D_{r}\right) * p_{g} * p_{a} /\left(1-p_{g} * p_{a}\right)
\end{aligned}
$$

where $p_{g}$ is the surface reflectance, the value is $0.2 ; p_{a}$ is the clear sky retroreflectivity, the value is $0.0685, \tau_{0}$, $\tau_{g}, \tau_{r}$ are respectively the absorption ratio of ozone absorption, carbon dioxide, oxygen, etc. Air molecular diffuseing transmittance, all three parameters are a function of $M_{h}$.

\subsubsection{Calculation of reflected radiation from the wall of adjacent buildings}

The reflection of the building wall against direct solar radiation and diffuseed radiation, this article ignores multiple reflections between the walls of the building, considering only one reflection.

According to the simplified approximate calculation method of Dozier [24], only the sky view factor and the wall average reflection are considered. The calculation formula for the reflected radiation from the wall of adjacent buildings is:

$$
I_{r}=p_{\text {wall }} *(1-S V F) *\left(I_{b}+I_{d}\right)
$$

where $p_{\text {wall }}$ is the average reflectivity of the wall, and its empirical value is 0.22 ; SVF is the openness of the sky. 


\subsubsection{Calculation of Total Solar Radiation}

The solar radiation reaching the surface is divided into two types: direct solar radiation and sky-diffuseed radiation. In addition, there is solar radiation from the surrounding reflection. Therefore, the solar radiation incident on the ground is the direct solar radiation, the diffuseed radiation and the reflected radiation from the wall of the adjacent building, called the total solar radiation of the ground. When a point on the ground is covered by the shadow of the building at a certain moment, the total amount of solar radiation is the diffuseed radiation and the reflected radiation from the adjacent building wall. The formula for calculating the total solar radiation is:

$$
\begin{aligned}
& \mathrm{I}=I_{b}+I_{d}+I_{r} \\
& \mathrm{I}=I_{d}+I_{r}
\end{aligned}
$$

where in Equation 11 is the total amount of solar radiation under unobstructed conditions, and Equation 12 is the total amount of solar radiation when there is occlusion.

\subsection{Verification Algorithm and Modeling Method}

This paper compares the measured solar radiation data of actual urban settlements with the calculated data of solar radiation calculation model based on SVF, and tests the computational performance of the algorithm. Simplify the building during modeling, build a simplified building model based on the building profile, and ignore the detailed components (such as roofing members, bay windows, lumps, legs, etc.). Simplified residential model as shown (Fig. 3)

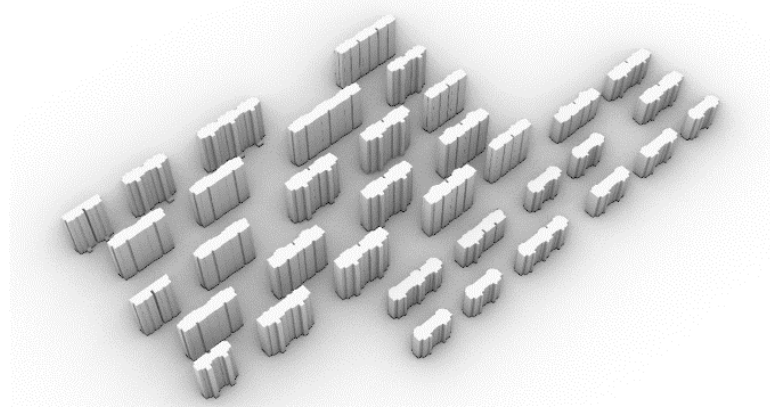

Fig. 3. Simplified model in Rhino.

\section{Results and discussion}

Based on the RH \& GH parameter platform, the SVF calculation results are shown in Fig. 8. Since the measuring points 1,5 and 12 are close to the building, they are blocked by the building, and the SVF is small, 0.31, $0.38,0.37$, respectively.4, 11,17 are more open, SVF is larger, $0.64,0.60,0.60$.

The total solar radiation value of the settlement is selected from the hourly data during the test, and the measured data is analyzed. The total solar radiation dose of the four measuring points not covered by the shadow is consistent throughout the day (Fig.4), from 09:00 to noon. The total amount of solar radiation reaching the measuring point gradually increases; from noon to $18: 00$, the total solar radiation gradually decreases until it reaches zero. The maximum occurs at the measuring point 11 , and the SVF at this point is 0.60 , which is also the largest of the four measuring points. At the point covered by the shadow, the total amount of solar radiation will decrease with the coverage of the shadow.

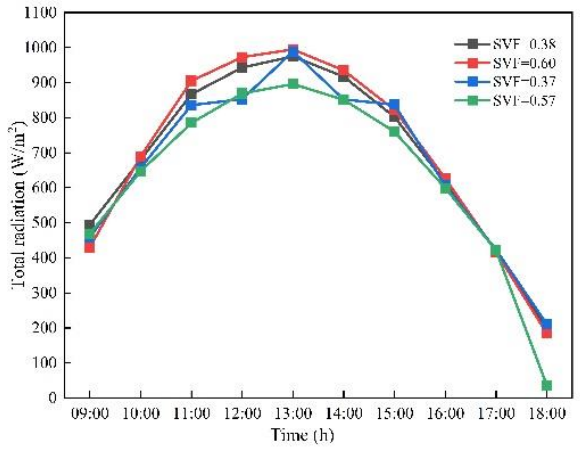

Fig. 4. Total solar radiation value at unobstructed points throughout the day.

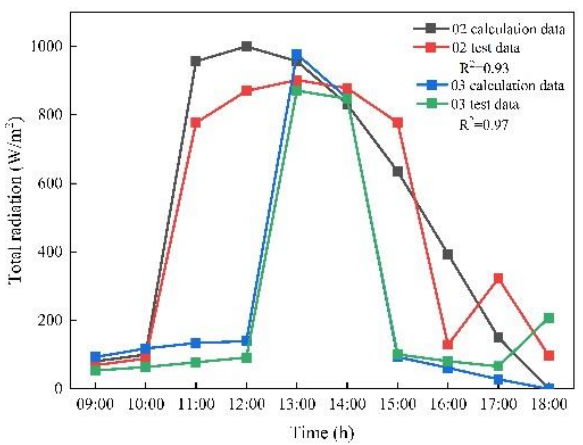

Fig. 5. Comparison of the total radiation values of the calculation and test of the measuring point 02 and 03

The calculated data using the calculation model is compared with the measured data. The calculation result of the total solar radiation is in good agreement with the test results. The calculation method of the $d$ index of the index of agreement (d value) is as follows (Equation 13):

$d=1-\frac{\sum_{i}^{n}\left(x_{c_{i}}-x_{m_{i}}\right)^{2}}{\sum_{i}^{n}\left(x_{c_{i}}+x_{m_{i}}\right)^{2}} \quad 0 \leq \mathrm{d} \leq 1$

where $x_{c_{i}}, x_{m_{i}}$ are the calculated data and the measured data respectively. Taking the measuring point 02,03 as an example (Fig. 5), it changes with time during the daytime. The curve is consistent. Correlation coefficient and consistency index are introduced, and the calculated data and measured data are used. The correlation coefficient between them shows the correlation coefficient between the measured total solar radiation and the calculated data of 18 measuring points (Fig. 6). The coefficient is the largest, reaching 0.99 . The correlation coefficient of measuring point 1 is the smallest, the correlation coefficient is 0.79 . Most of the measured points have a correlation coefficient of about 0.9. The reason for 
analyzing the correlation coefficient of measuring point 1 is small. It is found that the test value at 14:00 is too small, and the correction is corrected. After the value, the correlation coefficient $\mathrm{R}^{2}$ reaches 0.99 . The consistency index between the calculated data and the measured data,except for the $d$ value of the measuring point 1 is 0.94 the $\mathrm{d}$ values of the other measuring points are all around 0.98 .

In order to understand the diffuseed radiation in the residential area, the calculation model is used to calculate the solar diffuse values of different measuring points(Fig.7). As can be seen from the figure, the solar

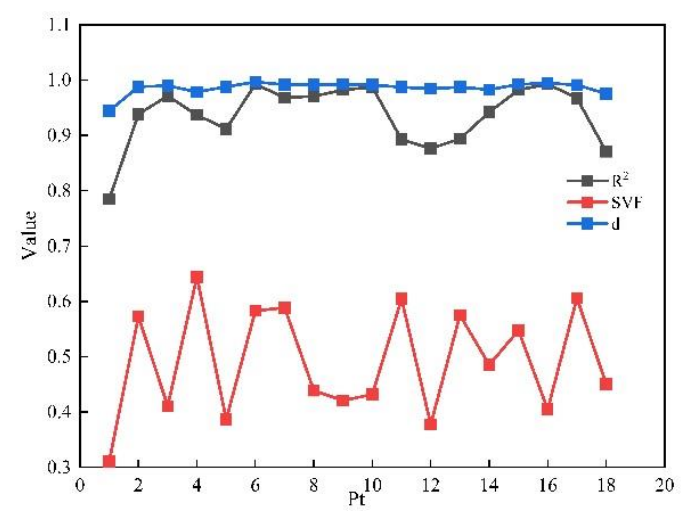

Fig. 6. Correlation coefficients and index of agreement for each measurement point.

diffuse of all measuring points changes with time, from 09:00 to noon, reaching the measuring point. The amount of solar diffuseed radiation gradually increases; from noon to 18:00, the amount of diffuseed radiation decreases again. The minimum value appears at 18:00, which is basically zero, and the maximum value appears at noon. For all measuring points, the amount of solar diffuse radiation is less than $34.90 \mathrm{~W} / \mathrm{m}^{2}$ during the daytime, which is much smaller than the direct solar diffuse. At the same time, the difference in solar diffuseing at different measuring points is due to the difference in SVF, and the large diffuseing value of the SVF value is large.

In order to understand the reflected radiation in the residential area, the calculation model is used to calculate the wall reflection radiation of different measuring points. It can be seen from the figure that the reflection values of all measuring points change with time, from 09:00 to noon, reaching the measuring point (Fig. 8). The reflected radiation from the wall gradually increases, from noon to 18:00, the reflected radiation gradually decreases, and the minimum appears at $18: 00$, which is basically 0 , and the maximum appears at noon. For all measuring points, the reflected radiation is less than $138.38 \mathrm{~W} / \mathrm{m}^{2}$ during the daytime period. Although it is larger than the amount of diffuseed radiation, it is still much smaller than the direct diffuseing amount. At the same time, the measuring point with large SVF value is slightly smaller.

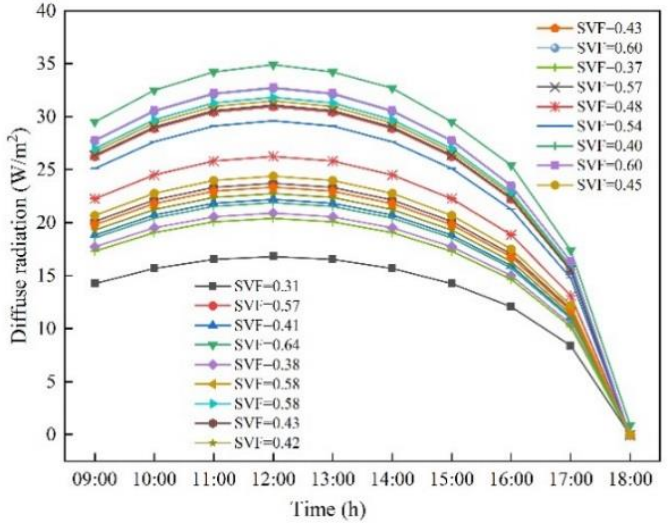

Fig.7. Calculate the diffuse radiation of the measured point using a computational model.

\section{Conclusions}

Based on the ladybug plugin and through the scripting language, the Rhino \& Grasshopper parameterization platform is used to realize the automatic calculation of the sky angle factor. Comparing the measured data of solar radiation with the calculated data of solar radiation calculation model based on SVF, the correlation coefficient of the two groups of data is about 0.9 , and the consistency index $d$ value is about 0.98 , which indicates that the algorithm has higher sky angle factor. The calculation accuracy and stability, and the selected SVFbased solar radiation model also has higher progress and stability. This paper provides a faster and faster parameterization method for calculating solar radiation of the three-dimensional model.

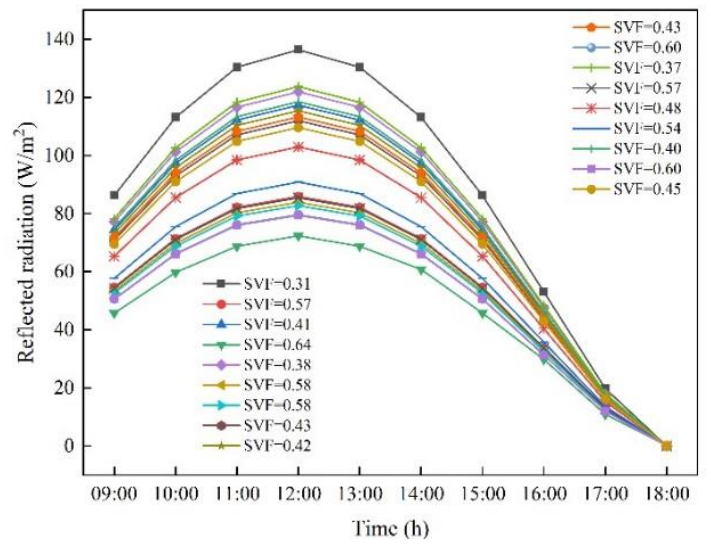

Fig.8. Calculate the reflected radiation of the measured point using a computational model.

\section{Acknowledgement}

This work was supported by National Natural Science Foundation of China (No. 51838011 and No. 51808429), Young Elite Scientists Sponsorship Program by CAST, YESS (No. 2018QNRC001).

\section{References}

1 http://www.prb.org/Publications/Datasheets (2015) 
2 C. Chatzipoulka, R. Compagnon, M. Nikolopoulou. Solar Energy. 138, 53-66 (2016).

3 OKE.T R Boundary layer climates. Second Edition . Outledge. 40, 17-26 (1988)

4 C. Chatzipoulka, R. Compagnon, J. Kaempf, M. Nikolopoulou. Solar Energy. 170, 1026-1038 (2018).

5 W. Li, S.Y. Putra, P. J. Yang. GISDECO. (2004).

6 http://www.urbanclimate.net/rayman/ (2010)

7 I. D. Watson, G.T. Johnson. Jourl. Climat. 7, 193-197. (1987)

8 G. Johnson, J. Waston. Jourl. Climate. Appli. Mete. 23, 329-335 (1984)

9 C. Grimmond, S. Potter, H. Zutter, et al. Inter. Jourl. Climat. 21, 903-913 (2001)

10 D. Steyn. Jourl. Atmos. Ocean. Tech. 18, 254-258 (1980)

11 L. Chapman, J. Thornes. Jourl. Atmos. Ocean. Tech. 21, 720-742. (2004)

12 J. Unger, Matzarakis. Build. Environ. 41, 1713-1722 (2006)

13 C. Ratti, P Richard, Proceed. CAAD. Futures. (1999)

14 B. Li, Q. Luo, R. Yao. Jourl Chongqing University 29, 86-89. (2006)
15 J. Wu, Y Zhang, Q. Meng. 13th Conference of International Building Performance Simulation Association. (2013)

16 E. Bazafkan. Assessment of Usablity and Usefulness of New Building Performance Simulation Tool in Architectural Design Process.Vinenna University of Technology. (2017)

17 https://www.ladybug.tools/epwmap/

18 H Zhang. Study of Urban Thermal Environment Based on Remote Sensing and Urban Spatial Morphology. (2015)

19 D. Zuo. Spatial distribution characteristics of total solar radiation in China. Jour. Mete. 33, 78-95. (1963)

20 G. Gao Meteorology course. (1996)

21 Y. Zhao. Principles and methods of remote sensing application analysis. Beijing (2003)

22 F. Kreith, J Kreider. Principle of Solar Engineering. McGraw-Hill. (1978)

23 M. Iqbal. An introduction to solar radiation. Aca. Pre. (1983)

24 J. Dozier, J. Frew. IEEE Trans. on Geosci and Rem.Sen. 28, 963-969. (1990) 